\title{
Analizando el PIB a la luz de la Teoría de las Capacidades de Martha Nussbaum Analysing GDP in the light of Martha Nussbaum's Capabilities Theory
}

\author{
Cristina Vilaplana Prieto \\ cvilaplana@um.es
}

\author{
Departamento de Fundamentos de Análisis Económico \\ Universidad de Murcia \\ Murcia, España
}

\begin{abstract}
Resumen- Resulta difícil medir el progreso económico. A pesar de su amplia utilización, el PIB constituye una medida insuficiente del bienestar, ya que el progreso económico no garantiza la mejora del desarrollo humano. Entre las muchas alternativas propuestas, destaca la "Teoría de las Capacidades" de Martha Nussbaum (Premio Príncipe de Asturias de las Ciencias Sociales, 2012). En este artículo se explica cómo se ha introducido dicha teoría para la realización de un proyecto de innovación docente dentro de la asignatura de "Principios de Economía" del Grado en Ciencias Políticas y Gestión Pública. El objetivo es que los estudiantes relacionen economía y crecimiento económico con justicia social, sostenibilidad e igualdad.
\end{abstract}

Palabras clave: economía, PIB, capacidades, desarrollo, crecimiento

Abstract- It is difficult to measure economic progress. In spite of its great predominance, the GDP constitutes an insufficient well-being measure, because economic progress does not guarantee the improvement of human development. Among the many alternatives proposed, it is worth to highlight the "Theory of Capabilities" by Martha Nussbaum (Prince of Asturias Award for Social Sciences, 2012). This article explains how this theory has been applied in an innovation teaching project of the subject "Principles of Economy" in the "Grade of Political Science and Public Management". The aim is that students relate economics and economic growth to social justice, sustainability and equality.

Keywords: economy, GDP, capabilities, development, growth.

\section{INTRODUCCIÓN}

Las universidades desempeñan un papel especial en la sociedad a través de sus actividades principales de fomento del conocimiento y la erudición y de formación de estudiantes y profesionales. Sin embargo, en la época contemporánea, la educación superior se presenta más comúnmente como el escenario clave para transformar un país en una economía competitiva basada en el conocimiento, capaz de combinar el crecimiento económico y la mejora del empleo con una mayor cohesión social. Nussbaum (2010) describe esta situación como la insaciable sed de crecimiento económico y resultados de capital humano que impulsa la política educativa en todo el mundo. la pregunta más importante. ¿Por qué la intuición básica de que las preocupaciones humanas deben ser el objetivo último de la actividad económica sigue siendo ignorada en la política de todo el mundo?
No es que el capital humano sea un mal objetivo para las universidades: las oportunidades económicas justas, un entorno empresarial productivo y la reducción de la inseguridad humana son fundamentales para el bienestar. La integración en la vida económica es importante para que las personas participen plenamente en la generación de ingresos y en la obtención de un empleo remunerado. Pero los ingresos por sí solos no pueden captar toda la gama de contribuciones a un estado de bienestar en la vida de una persona (Sen, 1999).

El enfoque de la capacidad de Amartya Sen (1999) y su desarrollo posterior por Nussbaum $(2000,2010)$ ofrecen un enfoque normativo atractivo al subsumir el capital humano dentro de un enfoque que valora el bienestar humano, y en el que cada persona es un fin en sí mismo y no el medio para algún otro fin. La educación universitaria puede contribuir a lo que Sen (1999) denomina "funcionamientos" (logros), es decir, aquellos seres y hechos valiosos que nos permiten elegir y vivir de maneras que encontramos significativas, productivas y gratificantes individual y colectivamente para el bien de la sociedad. Para Sen, las capacidades son las libertades, los potenciales o los caminos vitales alcanzables para que un individuo logre un funcionamiento valioso, de modo que "el conjunto de capacidades nos da información sobre los diversos vectores de funcionamiento que están al alcance de una persona" (Sen, 1992, pp. 41-42). Los funcionamientos son constitutivos del bienestar y la calidad de vida de una persona y su ausencia sugiere malestar y desventaja.

Sen (2009) rechaza firmemente la opinión de que una vida mejor sólo puede derivarse del crecimiento económico: hay una serie de fines humanos valorados, de modo que ser un mejor productor no es el único fin evaluativo para las vidas humanas; el propósito clave del desarrollo, sostiene, es el desarrollo humano. Los ingresos y la economía seguirían siendo importantes, pero el propósito del desarrollo educativo sería ampliar todas las opciones humanas que merecen la pena. Además, Nussbaum (2011) destaca explícitamente el valor de la dignidad humana. Para Nussbaum (2000, 2011) las capacidades son oportunidades para pensar en el currículo, y "poderes personales". Nussbaum propone una personalidad más compleja que la de Sen, con su lista de diez capacidades universales centrales que dan contenido a la noción abstracta de 


\section{ANALIZANDO EL PIB A LA LUZ DE LA TEORÍA DE LAS CAPACIDADES DE MARTHA NUSSBAUM2}

capacidad. Si hacemos que las capacidades y los funcionamientos sean centrales en el currículo, cambiamos el eje de análisis para establecer y evaluar las condiciones políticas, sociales, económicas y educativas que permiten a los individuos tomar decisiones informadas, basadas en lo que tienen razones para valorar.

El enfoque de las capacidades se basa en una distinción fundamental entre capacidades y funcionamientos, donde una capacidad es la libertad real que tiene un individuo para funcionar de una manera que valora, mientras que un funcionamiento es hacer o ser algo. El énfasis principal dentro del enfoque está en las capacidades, ya que las personas no deberían (salvo en circunstancias excepcionales) verse obligadas a funcionar en contra de sus propios valores y/o elecciones, aunque otra persona lo considere deseable. Se puede argumentar que los Objetivos del Desarrollo Sostenible (ODS) son una versión de hacer o ser, en el sentido del enfoque de las capacidades. En este sentido, también se puede argumentar la necesidad de hacer hincapié en las capacidades, más que en los funcionamientos, en los individuos y las sociedades para promover los ODS (ONU, 2015; Sanz et al., 2018).

Este planteamiento engarza con la Educación para la Justicia Social que reclama una educación que trabaje en el desarrollo integral de los estudiantes, una educación crítica, que se pregunte explícitamente el porqué de las cosas para luchar contra las injusticias y opresiones (Murillo y HernándezCastilla, 2014).

\section{CONTEXTO}

En el curso académico 2010/2011 comenzó a impartirse el "Grado en Ciencias Políticas y Gestión Pública" (GCPGP) de la Universidad de Murcia. Este grado surgió como una fusión de las anteriores Licenciatura en Ciencias Políticas y Diplomatura en Gestión y Administración Pública. En el primer cuatrimestre del primer año se imparte la asignatura de "Principios de Economía" con una carga docente de 6 créditos, lo que equivale a tres horas de teoría y una hora de prácticas semanales.

Después de varios años impartiendo esta asignatura, se ha percibido la necesidad de introducir algún ejercicio que invite a los estudiantes a reflexionar y desarrollar un pensamiento crítico, y que también demuestre que la Economía está totalmente relacionada con otras áreas de conocimiento que comparten docencia en el primer cuatrimestre del GCPGP, como Sociología, Políticas Públicas, Instituciones Políticas, Historia y Filosofía Política. La concesión del "Premio Príncipe de Asturias de las Ciencias Sociales" en octubre de 2012, a la profesora Martha Nussbaum ha constituido el punto de partida para introducir una innovación docente.

El contexto en el que se imparte la asignatura de "Principios de Economía" posee unas particularidades especiales: (i) es una asignatura con un cierto carácter numérico que comparte docencia en el mismo cuatrimestre con otras asignaturas con un carácter mucho más teórico (Sociología, Derecho Constitucional, Fundamentos de Ciencia Política, Historia Política y Social Contemporánea); (ii) un porcentaje sustancial de los estudiantes asocian la economía con las matemáticas, y no han estudiado matemáticas en los dos años de bachillerato. Por estos motivos, se considera que es necesario que los estudiantes perciban que los datos, los resultados de los problemas numéricos o la interpretación de las representaciones gráficas son sólo instrumentos sobre los que sustentar el análisis crítico y la toma de decisiones de política económica.

Cuando se comparan varios países entre sí, o la trayectoria de un país a lo largo del tiempo, se suele identificar un mayor desarrollo con un mayor PIB per capita o un mayor crecimiento del mismo. Sin embargo, la utilización del PIB per capita puede "oscurecer" otros aspectos importantes que deben ir asociados al desarrollo (salud, educación, participación política, igualdad de género, libertad de asociación, libertad de elección de ocupación...). En palabras de ul Haq (1995; p.46), y en referencia a la forma de contabilizar del PIB, "cualquier medida que valore un revólver varios cientos de veces más que una botella de leche suscita serias dudas sobre su relevancia para medir el progreso humano".

Uno de los primeros indicadores alternativos al PIB fue el Índice de Desarrollo Humano (IDH o Human Development Index) en el año 1990, elaborado por el economista pakistaní Mahbub ul Haq, junto con un grupo de intelectuales entre los que se encontraba Amartya Sen (Premio Nobel de Economía en 1998). El IDH toma como punto de partida el Informe sobre Desarrollo Humano de Naciones Unidas (1990) y considera que existen tres necesidades básicas que deben ser cubiertas (conseguir una vida larga y saludable, adquirir conocimientos y disponer de los recursos necesarios para disfrutar de una calidad de vida decente) (UNDP, 1990).

A pesar de su buena intención, el IDH omite otras dimensiones relevantes (equidad, libertad política, derechos humanos, sostenibilidad, felicidad, por citar sólo unos cuantos). Por esta razón, pocos años después de su nacimiento empezaron a surgir una serie de críticas en torno a la limitada perspectiva de dicho índice. Por ejemplo, Dasgupta y Weale (1992) intentaron re-elaborar el IDH incluyendo variables relacionadas con la libertad civil y política; Sagar y Najam (1998) señalaron que el IDH no tenía en cuenta el efecto del desarrollo de los países sobre el mediambiente. Más recientemente, Martha Nussbaum $(2000,2003)$ enunció su teoría de las capacidades, la mayoría de las cuales no encuentran cabida en el IDH, pero permiten obtener una visión más global y realista del progreso humano.

\section{DESCRIPCIÓN}

La gran aportación de Martha Nussbaum es la configuración de una lista de capacidades fundamentales, es decir, una lista de principios básicos sin los cuales ninguna sociedad puede llamarse a sí misma "justa", independientemente de cuál sea su nivel de opulencia. Puede que la utilización del término "capacidades" resulte un poco llamativo al principio, y que se prefiriera denominarlos "derechos". No obstante, en este aspecto Martha Nussbaum aclara que la lista que elabora es de "capacidades" y no de "derechos", puesto que un derecho puede existir porque el mismo esté recogido en un texto legal, a pesar de que no existan medidas efectivas que permitan a las personas ser capaces de ejercitar dicho derecho.

Un ejemplo claro que rubrica esta diferenciación lo encontramos en la Constitución de Pakistán ${ }^{i}$, donde se establece que: “a) Todos los ciudadanos son iguales ante la Ley y deben recibir igual protección; b) No existirá discriminación por razón de sexo" (art. 25) y "El Estado promoverá con especial cuidado la educación en las clases sociales y en las áreas geográficas más desfavorecidas, promoverá la educación gratuita y obligatoria hasta la enseñanza secundaria y la 
educación superior sea accesible a todos sobre la base del mérito" (art. 37; Constitution of the Islamic Republic of Pakistan, 2018). Por consiguiente, se reconoce el derecho a la igualdad y el derecho a la educación. No obstante, todos conocemos el caso de la niña pakistaní, Malala Yousafzai, que recibió un disparo en la cabeza, simplemente por defender el derecho de las niñas pakistaníes a ir a la escuela, es decir, que las niñas no tenían la capacidad de educarse en igual condiciones que los niños.

Esta lista de capacidades se refiere a un conjunto de cuestiones relativas al ser humano que son verdaderamente trascendentes y que no se pueden dejar al albedrío de cada país, sino que deben ser reconocidas a nivel mundial A continuación, se exponen las diez capacidades propuestas por Martha Nussbaum. En su obra no se concreta cómo medir cada una de dichas capacidades, por lo que para poder llevarlas a la práctica ha sido necesario realizar una búsqueda de indicadores apropiados a cada una. Aunque se ha realizado una búsqueda exhaustiva que comprende un total de 48 indicadores, no se trata de una lista cerrada, sino que es susceptible de poder ampliarse. En cualquier caso, tampoco se ha querido saturar a los estudiantes con una lista de variables excesivamente larga.

En el Apéndice se detalla la traducción al inglés de cada indicador y la fuente donde se pueden encontrar los últimos datos disponibles. Esta información detallada fue suministrada a los estudiantes para que la utilizaran como guía en su búsqueda. Todas las páginas web que deben consultar los estudiantes están en inglés, por lo que la realización del trabajo constituye una forma de que fomentar el auto-aprendizaje de una lengua extranjera. Por otra parte, la recopilación de la información exige visitar páginas web de numerosas instituciones internacionales, y constituye una forma de familiarizarse con la labor de las mismas (Banco Mundial, Global Footprint Network, International Labor Organization, OCDE, Transparency International, UNICEF, United Nations).

1. Vida:

Ser capaz de gozar de una vida de duración normal (evitar la muerte prematura). Aunque a nivel individual, la "capacidad" relacionada con la "vida" se mide por la esperanza de vida al nacimiento, a nivel de una sociedad, también se debe tener en cuenta la relación entre la población en edad de trabajar y la población dependiente (menores de 15 años o mayores de 65 años).

\section{Salud corporal:}

Ser capaz de disfrutar de buena salud, estar adecuadamente nutrido y poder tener un lugar donde albergarse. Las variables que se han considerado como indicadores de esta capacidad se refieren tanto a estado de salud (mortalidad infantil y maternal, malnutrición), como a la disponibilidad de recursos para satisfacer las necesidades básicas.

\section{Integridad corporal:}

Ser capaz de moverse libremente, con seguridad, sin sufrir violencia sexual o violencia doméstica. Las variables que reflejan esta capacidad se refieren a situaciones en las que la mujer (niña o adulta) es víctima de maltrato y/o agresión.

\section{Imaginación y pensamiento:}

Ser capaz pensar, razonar y poder expresarse con libertad en el ámbito artístico, político y religioso. Poder recibir una educación que desarrolle las habilidades lingüísticas, matemáticas y el pensamiento científico.

\section{Emociones:}

Ser capaz de poder expresar sentimientos, sin que la exteriorización de los mismos se vea frustrada por el miedo o la intranquilidad. Las variables que se utilizan miden tanto la satisfacción con la vida (esperanza de años de felicidad) como la infelicidad (suicidio y bullying).

\section{Razón práctica:}

Ser capaz de reflexionar y planificar lo que se quiere realizar en la vida, incluyendo la protección a la libertad de conciencia y la observancia religiosa. Las variables que denotan esta capacidad se refieren tanto a la posibilidad de que la persona pueda realizarse en su faceta laboral, social y religiosa.

\section{Afiliación}

Esta capacidad engloba otras dos: (1) ser capaz de vivir e interaccionar con otras personas, lo que implica a su vez, proteger la libertad de asamblea y la libertad de discurso político y (2) no discriminación por razón de sexo, orientación sexual, raza/etnia, sexo, religión o nacionalidad.

\section{Relación con la naturaleza:}

Ser capaz de vivir respetando la naturaleza. Se utilizan indicadores que miden la contaminación y el deterioro del medio ambiente a causa del hombre.

\section{Ocio:}

Ser capaz de disfrutar de actividades de ocio y esparcimiento. Se propone utilizar como indicadores determinados la presencia de determinados comportamientos fruto de un ocio mal entendido (consumo de alcohol en los jóvenes, población con sobrepeso), así como la ausencia de tiempo de ocio (niños que están trabajando en lugar de disfrutar de su infancia, mujeres que son obligadas a casarse a edad temprana).

\section{Control del entorno:}

Esta capacidad engloba otras dos: (1) participar efectivamente en los procesos políticos y (2) ser capaz de tener una propiedad, de buscar empleo en las mismas condiciones que otros y ser capaz de ejercer una profesión con dignidad y con reconocimiento por parte de otros.

\section{A. Planteamiento de la actividad}

Al estudiar el concepto de Producto Interior Bruto (PIB), los estudiantes comprueban que hay muchas noticias del día a día en el que se utiliza esta magnitud: déficit y deuda pública respecto al PIB, tasa de crecimiento del PIB como indicador de fase del ciclo económico o PIB per capita. Sin embargo, el cálculo del PIB presenta una serie de limitaciones:

1. No registra las transacciones llevadas a cabo en términos de "economía sumergida" o en "dinero negro".

2. No se registran las operaciones de "autoconsumo", "trueque" y demás actividades no retribuidas formalmente.

3. La valoración de la actividad económica no considera la "calidad" de la producción, ni la "eficiencia" con la que se generan los bienes y servicios.

4. Omisión de "costes medioambientales" en concepto de destrucción de recursos naturales.

5. Los organismos encargados de elaborar la Contabilidad Nacional tienen que enfrentarse a numerosos problemas técnicos lo implica que para la obtención de cifras definitivas es necesario que transcurra bastante tiempo.

Como colofón a estas manifestaciones sobre la interpretación con reservas de los indicadores basados en el PIB, 


\section{ANALIZANDO EL PIB A LA LUZ DE LA TEORÍA DE LAS CAPACIDADES DE MARTHA NUSSBAUM4}

el Senador Robert Kennedy, en su discurso a las elecciones presidenciales de 1968 señalaba que: "el PIB no tiene en cuenta la salud de nuestros niños, ni la calidad de su educación. No mide ni nuestro coraje ni nuestra sabiduría ni nuestra devoción a nuestro país. Lo mide todo, salvo lo que hace que la vida merezca la pena" (JFK Library, 1968; p.5).

A continuación, se planteó a los estudiantes la posibilidad de que fueran ellos mismos los que determinaran si el nivel de progreso económico (medido por el PIB) estaba de acuerdo con el nivel de bienestar, mediante la realización de un trabajo en equipo.

\section{B. Formación de grupos de trabajo:}

Se formaron grupos de 3 ó 4 estudiantes (dando como resultado 32 grupos). Cada grupo de trabajo debía analizar las capacidades de Marta Nussbaum para un grupo de países. Los estudiantes no seleccionaban el grupo de países que iban a estudiar, sino que hizo por sorteo, utilizando la clasificación de países del Fondo Monetario Internacional (Fiscal Monitor, 2012). La clasificación de países del Fiscal Monitor divide el mundo en tres grandes grupos: 39 economías avanzadas, 96 economías de mercado emergentes y de renta media, y 59 países en desarrollo de renta baja.

\section{Contenido del trabajo:}

El objetivo del trabajo es invitar a los estudiantes a que exploren otras formas de medir el desarrollo de un grupo de países. El punto de partida es la "Teoría de las capacidades" propuesta por Martha Nussbaum. El material relativo a la lista de capacidades de Martha Nussbaum, las variables propuestas para medir cada una de ellas y la bibliografía sobre otros autores que han abordado este tema se encontraba disponible en el "Sitio de la asignatura" dentro del Aula Virtual.

Se han realizado sesiones de tutorías grupales para comentar la evolución del trabajo, el funcionamiento del grupo y resolución de dudas. En estas tutorías grupales se han reunido simultáneamente los miembros de 4 grupos de trabajo diferentes, y aunque son los propios estudiantes los que deben aprender a relacionar los objetivos con los indicadores estadísticos correspondientes, las tutorías sirven para orientarles en este proceso de traslación.

El trabajo de los estudiantes consistió en:

(1) Recopilar información sobre cada uno de los indicadores para la lista de países que se les hubiese asignado

(2) Mostrar la información de forma ordenada

(3) Comparar el PIB per capita (en paridad del poder adquisitivo) con la lista de capacidades y con el Índice de Desarrollo Humano

(4) Reflexionar sobre las siguientes cuestiones: ¿son los países con mayor PIB per capita los que también presentan los valores más elevados de los otros indicadores? O por el contrario, ¿hay algún país de los estudiados que tiene menor PIB per capita, pero destaca en algún otro indicador reflejo de alguna de las capacidades analizadas?

\section{Evaluación:}

El trabajo representa un $20 \%$ de la nota final (es decir, se evaluaba con una nota máxima de 20 puntos sobre 100). Los instrumentos utilizados en la evaluación son los siguientes: i. Presentación en clase de los resultados (10 minutos) y entrega del trabajo (hoja de cálculo y comentarios).

ii. Actas de las reuniones del grupo: éstas incluyen quiénes asistieron a cada reunión, las tareas completadas, las tareas planificadas y los contribuyentes a las distintas tareas. Esto proporciona al profesor una forma de monitorear las contribuciones individuales al grupo.

iii. Calificaciones de contribución individual y de grupo: la calificación del proyecto se divide en porcentaje de contribución individual y de grupo. Para valorar la contribución grupal se utilizó un sistema de rúbricas: (1) calidad del informe escrito (presentación y ortografia), (2) contenido del informe escrito (todos los países asignados y todos los indicadores para cada una de las capacidades), (3) análisis crítico de los resultados obtenidos. En relación a la contribución individual, cuando todos los estudiantes de un grupo contribuyen por igual al proyecto, todos obtienen la misma calificación de grupo, pero en ocasiones, un estudiante siente que ha que ha contribuido por encima del trabajo de cualquier otro en el grupo. Cuando esto ocurre, el estudiante que más ha trabajado puede proponer a su grupo que se le designe "autor principal" del trabajo y recibir la nota del trabajo más tres puntos de crédito extra. Todos los demás estudiantes son nombrados "autores colaboradores" del trabajo y reciben la nota tal cual. Si un estudiante realizó un trabajo mínimo según la evaluación del resto del grupo, se le considera un "reconocimiento" y recibe la nota menos cinco puntos. Los estudiantes que no contribuyen en absoluto reciben un cero. El grupo es el único responsable de la calificación diferencial, y lo demuestra asignando la autoría y firmando un documento para indicar su acuerdo. Si es necesario, el profesor puede actuar como mediador para ayudar a los estudiantes a resolver el conflicto, pero no determinará el resultado para el grupo.

\section{RESULTADOS}

La realización de esta actividad ha resultado muy satisfactoria tanto para los estudiantes, como desde un punto de vista personal. La realización de este trabajo es útil para el desarrollo de determinadas competencias:

- Competencias transversales de la Universidad de Murcia: (1) ser capaz de expresarse correctamente en español en su ámbito disciplinar; (2) ser capaz de gestionar la información y saber utilizar como usuario las herramientas básicas en TIC, (3) desarrollar habilidades de iniciación a la investigación.

- Competencias específicas del Grado en Ciencias Políticas y Gestión Pública: (1) análisis y síntesis, (2) orientación y planificación; (3) trabajo en equipo, (4) razonamiento crítico, (5) aprendizaje autónomo.

- Competencias específicas de la asignatura: (1) familiarizarse con el lenguaje, los conceptos, las herramientas y la lógica de la economía como forma de conocimiento, (2) capacidad de leer en inglés económicoempresarial y (3) analizar datos de investigación cuantitativos y cualitativos y operar con ellos.

\section{CONCLUSIONES}

La mayor parte de las prácticas que realizan en otras asignaturas consiste en la lectura y comentario de textos de 
carácter socio-politológico. Por este motivo, las principales ventajas de la realización de este proyecto de innovación docente son las siguientes: (i) los estudiantes aprenden a poner en valor la importancia de los datos estadísticos como fuente de información; (ii) los estudiantes extraen sus propias conclusiones a partir del análisis de dichos datos; (iii) los estudiantes son capaces de confrontar dichas conclusiones con la realidad predominante en la que se utiliza como indicador hegemónico el PIB. Adicionalmente, dado que esta signatura se desarrolla justo cuando los estudiantes acaban de iniciar la carrera también supone un refuerzo para el desarrollo de habilidades sociales. Este proyecto docente es fácilmente transferible a otros grados en los que la asignatura de "Economía" tiene una participación un tanto tangencial (Grado en Sociología, Grado en Relaciones Laborales y Recursos Humanos, Grado en Periodismo).

Entre las limitaciones hay que mencionar, que los escasos de conocimientos de Estadística (asignatura que se imparte en $2^{\circ}$ curso) ha impedido realizar un análisis más profundo de determinados aspectos como, por ejemplo, regresiones y gráficos de dispersión entre el PIB per capita y algún indicador para el grupo de países que tenían asignados.

Los estudiantes han manifestado que han aprendido mucho sobre las diferencias entre los países y que eso les ha ayudado a tomar una nueva conciencia de la sociedad en la que vivimos y de las diferencias respecto al modo de vida de otros jóvenes que viven otros países. El hecho de que no hubiera dos trabajos iguales ha ayudado a mantener el interés durante las exposiciones, puesto que siempre había nuevos aspectos socioeconómicos que suscitaban comentarios y reflexiones. Se espera que, en un futuro, este trabajo contribuya a que desarrollen un compromiso ético como valor esencial de su práctica profesional.

El objetivo de la educación debe ser proporcionar las condiciones curriculares y pedagógicas para que se desarrollen las capacidades de los futuros egresados. En cuanto a las actitudes $\mathrm{o}$ valoraciones afectivas a promover en los universitarios como gestores de la sostenibilidad, es indispensable generar una predisposición positiva a los valores humanistas. El modo en que finalmente se ejerzan dependerá de las decisiones de los individuos. Así, Nussbaum (2000, p. 88) escribe que "una vez que el escenario está totalmente preparado, la elección depende de ellos".

\section{REFERENCIAS}

Constitution of the Islamic Republic of Pakistan (2018). The Constitution of Pakistan (pakistani.org)

Dasgupta, P., Weale, M. (1992). On measuring the quality of life. World Development, 20(1), 119-131.

Fiscal Monitor (2012). IMF Fiscal Affairs Department. IMF Monitor 2012

JFK Library (1968). Remarks at the University of Kansas, March 15, 1968. Remarks at the University of Kansas, March 18, 1968 | JFK Library

Murillo, F. J. y Hernández-Castilla, R. (2014). Liderando escuelas justas para la Justicia Social. Revista Internacional de Educación para la Justicia Social, 3(2), 13-32.

Nussbaum, M. (2000). Women and human development: the capabilities approach. Cambridge: Cambridge University Press.
Nussbaum, M. (2003). Capabilities as fundamental entitlements: Sen and social justice. Feminist Economics, 9(2/3), 3359.

Nussbaum, M. (2010). Not for profit. Princeton: Princeton University Press.

Nussbaum, M. (2011). Creating capabilities. Cambridge, MA: The Belknap Press.

Sagar, A.D., Najam, A. (1998). The Human Development Index: a critical review. Ecological Economics, 25(3), 249-264.

Sanz, R., Ponce, J., Peris, A., Escámez, J. (2018). The capabilities approach and values of sustainability: Towards an inclusive Pedagogy. Journal of Innovation \& Knowledge, 3(2), 76-81.

Sen, A. (1992). Inequality re-examined. Oxford: Clarendon Press.

Sen, A. (1999). Development as freedom. Oxford: Oxford University Press

Sen, A. (2009). The idea of justice. London: Allen Lane.

Ul Haq, M. (1995). Reflections on human development. New York: Oxford University Press (pág. 46).

UN (2015). The sustainable development agenda. Disponible: https://www.un.org/sustainabledevelopment/developme nt-agenda/

UNDP (United Nations Development Programme) (1990). Human Development Report 1990. New York: Oxford University Press. (pág. 10).

\section{APÉNDICE}

\section{Vida}

1.1. Life expectancy at birth (years)

Fuente: Naciones Unidas (Human Development Report Office).

1.2. Age dependency ratio ( $\%$ of working age population) Fuente: Banco Mundial

\section{Salud corporal:}

2.1. Public expenditure on health (\% of GDP)

Fuente: Banco Mundial.

2.2. Income Gini coefficient

Fuente: Banco Mundial y OCDE Estadísticas.

2.3. Human Poverty Index (HPI)

Fuente: International Human Development Indicators y OCDE Estadísticas.

2.4. Maternal deaths per 100,000 live births Fuente: UNICEF.

2.5. Population living below $\$ 1.25$ (PPP) per day (\%) Fuente: Fuente: Naciones Unidas (Human Development Report Office).

2.6. Underweight (moderate and severe, \%) Fuente: UNICEF.

2.7. Under-five mortality rate. Fuente: Banco Mundial

\section{Integridad corporal}

3.1. Missing women

Fuente: OCDE Estadísticas.

3.2. Female Genital Mutilation Fuente: OCDE Estadísticas.

3.3. Violence against women

Se calcula a partir de la media de tres componentes: legislación, actitud frente a la violencia y prevalencia de la violencia doméstica. Fuente: OCDE Estadísticas. 


\section{ANALIZANDO EL PIB A LA LUZ DE LA TEORÍA DE LAS CAPACIDADES DE MARTHA NUSSBAUM6}

\section{Imaginación y pensamiento:}

4.1. Ratio of female to male in primary/secondary/tertiary education

Fuente: Banco Mundial.

4.2. Public expenditure on education (\% of GDP) Fuente: Banco Mundial.

4.3. Youth literacy rate (15-24 years) $(\%)$ Fuente: UNICEF. Disponible en: Learning and skills UNICEF DATA

4.4. Mean years of schooling (of adults)(years) Fuente: Naciones Unidas (Human Development Report Office).

\section{Emociones:}

5.1. Bullying Fuente: OCDE Estadísticas.

\subsection{Happy Life Expectancy} Fuente: World Database of Happiness"

5.3. Happy Planet Index Fuente: Happy Planet Index

5.4. Children suicide

Fuente: OCDE Estadísticas.

\section{Razón práctica:}

6.1. Ease of doing business

Fuente: World Bank Doing Business Project

6.2. Weighted Index of Social Progress (WISP)

Fuente: web de "Social Policy and Change" de la Universidad de Pennsylvania: Estes's Weighted Index of Social Progress (miqols.org)

6.3. Religious Freedom

Fuente: Bureau of Democracy, Human Rights and Liberty. Disponible "International Religious Freedom Report for 2019" (Departamento de Estado. Estados Unidos)

6.4. Discouraged workers

Fuente: International Labor Organization. ILOSTAT

\section{Afiliación:}

7.1. Minorities at Risk (MAR)

Fuente: Centre for International Development and Conflict Management. University of Maryland.

Disponible en MAR | Home (umd.edu)

7.2. Refugee population by country or territory of origin Fuente: United Nations High Commissioner for Refugees (UNHCR)

7.3. Shares in parliament, female-male ratio Fuente: Naciones Unidas (Human Development Report Office).

7.4. Voz política

Political voice. Se basa en dos componentes: (1) participación política (porcentaje de mujeres en el parlamento nacional en una escala de 0 a 1 , en donde 0 representa la igualdad) y (2) existencia de cuotas para promover la integración de las mujeres en el ámbito político. Fuente: OCDE Estadísticas.
8.1. PM10, country level (micrograms por cubic meter) Fuente: Banco Mundial.

8.2. Fertilizer consumption (kilograms per hectare of arable land). Fuente: Banco Mundial.

8.3. Carbon dioxide emissions per capita (tonnes) Fuente: Naciones Unidas (Human Development Report Office).

8.4. Threatened species (mammals, birds, reptiles, amphibians, fish, plants). Fuente: OCDE Estadísticas

8.5. Ecological footprint. Fuente: Global Footprint Network.

9. Ocio:

9.1. Drunkenness in teenagers/youth Fuente: OCDE Estadísticas.

9.2. Smoking in teenagers/youth Fuente: OCDE Estadísticas.

9.3. Mean weekly hours actually worked for total employment Fuente: International Labor Organization (ILO).

9.4. Population aged 15-19 (\%) ever married Fuente: United Nations Statistics. Women and men in families.

9.5. Percentage of children aged 5-14 engaged in child labour Fuente: UNICEF. Disponible en: https://data.unicef.org/resources/child-labour-2020 global-estimates-trends-and-the-road-forward/

9.6. Overweight or obese population ( $\%$ of total population) Fuente: OCDE Estadísticas.

10. Control del entorno:

10.1. Acceso al espacio público Access to public space Existencia de restricciones legales discriminatorias que impiden que las mujeres se desplacen libremente (elección de domicilio, visita a familiares o amigos, aprobación del marido para solicitar pasaporte). Fuente: OCDE Estadísticas.

10.2. Access to credit Igualdad de las mujeres frente a los hombres en el acceso al crédito. Fuente: OCDE Estadísticas.

10.3. Vulnerable female employment (\%) Fuente: Bando Mundial.

10.4.Corruption Perception Index Fuente: Transparency International.

10.5. Share of women in adult labor force Fuente: United Nation Statistics. Work.

10.6. Strictness of employment protection - collective dismissals. Fuente: OCDE Estadísticas en http://stats.oecd.org/. Labour/Employment Protection

10.7. Strictness of employment protection - regular employment. Fuente: OCDE Estadísticas.

10.8. Strictness of employment protection - temporary employment. Fuente: OCDE Estadísticas.

10.9. Women's wages relatives to men's Fuente: United Nation Statistics. Work.

\section{Medio ambiente}

